\title{
The Clinical Characteristics and Predictors of Refractory Mycoplasma pneumoniae Pneumonia in Children
}

\author{
Yuanyuan Zhang, Yunlian Zhou, Shuxian Li, Dehua Yang, Xiling Wu, Zhimin Chen* \\ Department of Pulmonology, Children's Hospital, Zhejiang University School of Medicine, Hangzhou, P.R. China \\ *drchenzm@163.com
}

\begin{abstract}
\section{Objective}

To analyze the clinical characteristics of refracory Mycoplasma pneumoniae pneumonia (RMPP), and explore the related factors predicting RMPP.
\end{abstract}

\section{Methods}

\section{Gopenaccess}

Citation: Zhang Y, Zhou Y, Li S, Yang D, Wu X, Chen Z (2016) The Clinical Characteristics and Predictors of Refractory Mycoplasma pneumoniae Pneumonia in Children. PLOS ONE 11(5): e0156465.

doi:10.1371/journal.pone. 0156465

Editor: Aldo Scarpa, University of Verona, ITALY

Received: November 19, 2015

Accepted: May 13, 2016

Published: May 26, 2016

Copyright: @ 2016 Zhang et al. This is an open access article distributed under the terms of the Creative Commons Attribution License, which permits unrestricted use, distribution, and reproduction in any medium, provided the original author and source are credited.

Data Availability Statement: All relevant data are within the paper.

Funding: This work was supported by grants from National Natural Science Foundation (81200022, $81401301,81501374)$. The funders had no role in study design, data collection and analysis, decision to publish, or preparation of the manuscript.

Retrospective analysis was performed on 634 children with Mycoplasma pneumoniae pneumonia (MPP) hospitalized in our hospital between January 1, 2011 and December 31, 2014. The clinical features, laboratory data, radiological findings between the RMPP group and the general Mycoplasma pneumoniae pneumonia (GMPP) group were compared and the predictive values of related factors were analyzed.

\section{Results}

The median age of the RMPP patients $(n=145)$ was much older than that of the GMPP patients $(n=489)(P<0.01)$. We also found more severe presentations, higher incidence of extra-pulmonary complications and more serious radiological findings in RMPP group, which needed oxygen more often, longer antibiotics administration and intensive care $(P<0.05)$. Meanwhile, the levels of $C$-reactive protein (CRP), lactic dehydrogenase $(\mathrm{LDH})$, immunoglobulin A (IgM), interleukin (IL)-6, IL-10, interferon gamma (IFN- $y$ ) and the percentage of neutrophils, CD8+ in RMPP group were significantly higher than those in GMPP group $(P<0.05)$; while the levels of prealbumin $(P A B)$ were lower than that in GMPP group $(P<0.01)$. In ROC curve analysis, the percentage of neutrophil, CRP, LDH, PAB, IL-6, IL-10 and IFN- $\gamma$ were useful for differentiating patients with RMPP from those with GMPP. Multiple logistic regression analysis showed that the CRP $\geq 16.5 \mathrm{mg} / \mathrm{L}, \mathrm{LDH} \geq 417 \mathrm{IU} / \mathrm{L}$ and IL-6 $\geq 14.75 \mathrm{pg} / \mathrm{ml}$ were significant predictors regarding to RMPP.

\section{Conclusions}

$\mathrm{CRP} \geq 16.5 \mathrm{mg} / \mathrm{L}, \mathrm{LDH} \geq 417 \mathrm{IU} / \mathrm{L}$ and $\mathrm{IL}-6 \geq 14.75 \mathrm{pg} / \mathrm{ml}$ might be the significant predictors of RMPP in children, which can aid in early recognition of RMPP.

Competing Interests: The authors have declared that no competing interests exist. 


\section{Introduction}

Mycoplasma pneumoniae (MP) is one of the most prevalent pathogens causing communityacquired pneumonia (CAP) in children [1,2]. Prior studies showed that MP might account for as many as $40 \%$ of CAP cases and $18 \%$ of these patients require hospitalization [3].

Although Mycoplasma pneumoniae pneumonia (MPP) is usually considered as a self-limited disease, sometimes it may cause various pulmonary and extra-pulmonary complications such as bronchiolitis obliterans, necrotizing pneumonia, encephalitis, arthritis, pericarditis, hemolytic anemia, and develop into a severe life-threatening pneumonia [4-11]. For children, macrolides are the first-choice antibiotics for MP infections. However, there still are some cases showing clinical and radiological deterioration despite of macrolide antibiotic therapy for 7 days or longer $[12,13]$ to be defined as refractory Mycoplasma pneumoniae pneumonia (RMPP). Therefore, it is important for clinicians to recognize RMPP earlier and grasp the appropriate opportunity for reasonable therapy.

In order to explore the predictive values of the independent related factors of RMPP, we retrospectively analyzed the cases of MPP hospitalized in our hospital between January 1, 2011 and December 31,2014, then compared the differences of clinical features, laboratory data and radiological findings between RMPP and general Mycoplasma pneumoniae pneumonia (GMPP) children.

\section{Methods}

\section{Study population}

In this study we retrospectively collected the data of patients with MMP who admitted to Children's hospital, Zhejiang University School of Medicine between January 1, 2011 and December 31,2014 . All the patients had signs and symptoms indicative of pneumonia on admission, including fever, cough, abnormal lung auscultation and a new infiltrate on chest radiograph [14]. The diagnosis of MP infection was based on the positive results for serologic test (MP IgM positive and antibody titer $\geq 1: 160$ ) while having the positive results for MP polymerase chain reaction (PCR) tests of nasopharyngeal secretions. The diagnosis of RMPP was based on the presence of persistent fever and clinical as well as radiological deterioration after azithromycin treatment for 7 days or longer $[12,13]$. All patients were excluded with other respiratory tract infections and tuberculosis by following tests: protein purified derivative (PPD), blood cultures, pleural effusion cultures, nasopharyngeal aspirate/swab cultures, nasopharyngeal aspirate/swab for virus antigens detection (respiratory syncytial viruses, influenza viruses, metapneumovirus, adenovirus, and parainfluenza virus), and serology for Chlamydia pneumoniae (CT) and Legionella pneumophila (LG). Patients who received corticosteroids before admission or had underlying diseases such as asthma, recurrent respiratory tract infection, chronic cardiac and pulmonary disease, rheumatic diseases and immunodeficiency were also excluded.

\section{Data collection}

Demographic, clinical information, laboratory data and radiological findings were retrospectively collected from all children who were included in the study. Nasopharyngeal aspirate/ swab specimens were routinely collected within 24 hours of admission. Respiratory specimens were tested for bacterial culture, virus using direct immunofluorescence assays and MP using PCR. Peripheral blood samples were obtained on admission for the determination of the complete blood count, C-reactive protein (CRP), lactate dehydrogenase (LDH), prealbumin (PAB), immunoglobulins, subpopulations of T lymphocytes, specific antibody to MP, CT, LG, and cytokines including interleukin (IL)-2, IL-4, IL-6, IL-10, tumor necrosis factor alpha (TNF- $\alpha$ ) 
and interferon gamma (IFN- $\gamma$ ). Blood culture was also performed on admission. The blood count and CRP were detected every 2-3 days thereafter if abnormal. The majority of patients were treated with macrolide (azithromycin or erythromycin) for 7-14 days, partly with a broad-spectrum antibiotic (ceftriaxone or amoxacilin) simultaneously. All patients underwent chest radiography before admission or during hospitalization, presenting unequivocal focal or segmental infiltration with or without pleural effusion. Large lesion was defined when the extent of infiltration on chest radiography was more than $1 / 3$ of the lung [15]. All children enrolled were followed until discharging. Body temperature and respiratory tract signs and symptoms of subjects were examined at study entry and every 8 hour thereafter. A febrile day was defined as a day during which the body temperature exceeded $38.0^{\circ} \mathrm{C}$ at least once [16]. During the hospitalization, we also evaluated the extra-pulmonary complications (liver function abnormalities, myocarditis, encephalitis, rash, proteinuria, hemolytic anemia and arthritis) of patients [17]. Hypoxia was defined as any recorded oxygen saturation of $<92 \%$ by pulse oximetry, measured on room air [18]. The indication for oxygen therapy and mechanical ventilation were evaluated according to the Guidelines for management of community acquired pneumonia in children in China [12].

\section{Ethics}

The study was approved by the ethics committee of the Children's Hospital, Zhejiang University School of Medicine. And the data from patients were analyzed anonymously.

\section{Statistical Analysis}

Statistical analyses were performed using SPSS software (version 15.0). Normal distribution data were expressed as mean $\pm \mathrm{SD}(\bar{x} \pm s)$. Independent-Samples T-test was used to compare these data. Skewed distribution data were expressed as median values (25th-75th interquartile ranges). The comparisons were made by the Mann-Whitney U-test. And Chi-squared tests were used to compare categorical data. Receiver operating characteristic (ROC) curves were operated to evaluate candidate indicators with regards to the refractory assessment of patients with MMP. Logistic regression analysis was performed to select the variables associated with the RMPP. Statistical significance was defined as $\mathrm{P}<0.05$.

\section{Results}

\section{General information of patients}

A total of 634 patients who were diagnosed with MPP in our hospital from January 1, 2011 to December 31, 2014 were enrolled in the study. All patients had positive results of PCR test and serological detection, and had not fulfilled exclusive criteria. The median age was 3.9 (2.1 6.8) years with a female-to-male ratio of 0.81 . All patients were previously healthy, without underlying disease. According to the diagnostic criteria of RMPP [12, 13], patients were divided into two groups, GMPP and RMPP. 489 patients were in the GMPP group (280 males, 209 females), with the median age of 3.4 (1.9 6.3) years. 145 patients were in the RMPP group (70 males, 75 females), with the median age of 5.9 (3.8 8.0) years. The median age of the RMPP patients was much older than that of the GMPP patients $(\mathrm{P}<0.01)$, but no difference in gender distribution was found between the two groups.

\section{Clinical and laboratory characteristics of GMPP and RMPP patients}

567 (89.4\%) patients presented fever in our study, but fever was more common in RMPP group than that in the GMPP group $(\mathrm{P}<0.01)$. We also compared other symptoms and 
Table 1. Clinical characteristic of GMPP and RMPP patients.

\begin{tabular}{|c|c|c|c|}
\hline Clinical information & GMPP $(n=489)$ & RMPP (n = 145) & $P$-value \\
\hline Age, years & $3.4(1.9 \sim 6.3)$ & $5.9(3.8 \sim 8.0)$ & 0.000 \\
\hline Sex (male/female) & $280 / 209$ & $70 / 75$ & 0.058 \\
\hline \multicolumn{4}{|l|}{ Clinical presentation, n (\%) } \\
\hline Fever & $422(86.3 \%)$ & $145(100 \%)$ & 0.000 \\
\hline Cough & $489(100 \%)$ & $145(100 \%)$ & 1.000 \\
\hline Tachypnea & $31(6.3 \%)$ & $35(24.1 \%)$ & 0.000 \\
\hline Chest tightness & $2(0.4 \%)$ & $4(2.8 \%)$ & 0.027 \\
\hline Wheezing & $70(14.3 \%)$ & $2(1.4 \%)$ & 0.000 \\
\hline Chill & $9(1.8 \%)$ & $9(6.2 \%)$ & 0.010 \\
\hline Extra-pulmonary complications & $63(12.9 \%)$ & $58(40.0 \%)$ & 0.000 \\
\hline \multicolumn{4}{|l|}{ Physical examination, n (\%) } \\
\hline Rales & $332(67.9 \%)$ & $86(59.3 \%)$ & 0.058 \\
\hline Decreased unilateral lung sound & $59(12.1 \%)$ & $60(41.4 \%)$ & 0.000 \\
\hline Length of fever, days & $7.0(3.3 \sim 10.0)$ & $13.0(11.0 \sim 16.0)$ & 0.000 \\
\hline Length of stay, days & $6.0(4.0 \sim 7.0)$ & $9.0(7.0 \sim 11.5)$ & 0.000 \\
\hline \multicolumn{4}{|l|}{ Management } \\
\hline Length of antibiotic therapy days & $11(8 \sim 11)$ & $16(14 \sim 18)$ & 0.000 \\
\hline Oxygenotherapy, n (\%) & $26(5.3 \%)$ & $40(27.6 \%)$ & 0.000 \\
\hline Intensive care unit, $\mathrm{n}(\%)$ & $0(0 \%)$ & $4(2.8 \%)$ & 0.003 \\
\hline
\end{tabular}

Data are presented as number (percentage), median $\left(25^{\text {th }}-75^{\text {th }}\right.$ percentile).

doi:10.1371/journal.pone.0156465.t001

respiratory signs between the two groups. The incidences of tachypnea, chest tightness, chill and decreased unilateral lung sound were significantly higher in the RMPP patients than those in the GMPP patients $(\mathrm{P}<0.01, \mathrm{P}<0.05, \mathrm{P}<0.05, \mathrm{P}<0.01$, respectively). The incidence of wheezing was significantly lower in the RMPP group than that in the GMPP group $(\mathrm{P}<0.01)$.

Of the 634 patients, extra-pulmonary complications were found in 121 cases (19.1\%), including digestive system in 28 , cardiovascular system in 29 , nervous system in 2, urinary system in 6 , joint system in 1 , rash in 50 , and hematological system in 5 . The incidence of extra-pulmonary complications was $40.0 \%$ in RMPP group and $12.9 \%$ in GMPP group, with a significant difference $(\mathrm{P}<0.01)$ (Table 1$)$.

Laboratory data and radiological findings in RMPP and GMPP patients were summarized in Tables 2 and 3. Regarding the laboratory examinations, the median levels of CRP, LDH, and the median percentage of peripheral neutrophils in children with RMPP were significant higher than those in children with GMPP $(\mathrm{P}<0.01)$; Patients with RMPP had significant lower level of $\mathrm{PAB}$, compared to that with GMPP $(\mathrm{P}<0.01)$. Meanwhile, we found significant higher levels of cytokines (including IL-6, IL-10, IFN- $\gamma$ ), IgA and higher percentage of CD8 ${ }^{+}$in the RMPP group when compared with the GMPP group ( $\mathrm{P}<0.01, \mathrm{P}<0.01, \mathrm{P}<0.05$, respectively). However, the median values of WBC, IgM, IgG, $\mathrm{CD}^{+}, \mathrm{CD}^{+}, \mathrm{IL}-2, \mathrm{IL}-4$ and TNF- $\alpha$ did not differ significantly between the two groups.

In addition to laboratory data, radiological findings were more severe in the RMPP group than that in the GMPP group (Table 3). 72.4\% of the patients in the RMPP group showed large lesions versus $34.4 \%$ in the GMPP group $(\mathrm{P}<0.01)$. And there were significant differences between the two groups in the incidence of pulmonary complications, including pleural effusion $(48.3 \%$ versus $11.0 \%, \mathrm{P}<0.01)$, lobar atelectasis $(26.2 \%$ versus $11.0 \%, \mathrm{P}<0.01)$, consolidation $(22.1 \%$ versus $3.9 \%, \mathrm{P}<0.01)$ and pleural thickening $(11.7 \%$ versus $3.5 \%, \mathrm{P}<0.01)$. But the 
Table 2. Laboratory characteristic of GMPP and RMPP patients.

\begin{tabular}{|c|c|c|c|}
\hline Laboratory information & GMPP $(n=489)$ & RMPP $(n=145)$ & $P$-value \\
\hline White blood cell $\left(\times 10^{9} / L\right)$ & $8.22(6.27 \sim 10.56)$ & $7.60(5.71 \sim 9.90)$ & 0.106 \\
\hline Neutrophil, \% & $56.7(43.4 \sim 65.6)$ & $73.1(65.3 \sim 78.5)$ & 0.000 \\
\hline C-reactive protein (CRP), mg/L) & $6(1 \sim 14)$ & $36(13 \sim 90)$ & 0.000 \\
\hline Lactatedehydrogenase (LDH), IU/L & 366 (310 459) & 537 (419 666) & 0.000 \\
\hline Prealbumin (PAB), g/L & $0.12(0.10 \sim 0.16)$ & $0.08(0.06 \sim 0.11)$ & 0.000 \\
\hline \multicolumn{4}{|l|}{ Total Immunoglobulin (Ig), g/L } \\
\hline $\lg G$ & $9.36(7.24 \sim 11.20)$ & $8.96(7.56 \sim 11.32)$ & 0.823 \\
\hline $\lg A$ & $0.85(0.51 \sim 1.31)$ & $1.20(0.77 \sim 1.54)$ & 0.000 \\
\hline $\lg M$ & $1.54(1.11 \sim 2.18)$ & $1.66(1.16 \sim 2.43)$ & 0.138 \\
\hline \multicolumn{4}{|l|}{ Subpopulations of T lymphocytes, \% } \\
\hline $\mathrm{CD}^{+}$ & $61.48(54.52 \sim 69.69)$ & $61.97(55.68 \sim 71.64)$ & 0.243 \\
\hline $\mathrm{CD}^{+}{ }^{+}$ & 33.97 (28.26 39.29) & $34.26(27.12 \sim 39.99)$ & 0.909 \\
\hline $\mathrm{CD}^{+}$ & $21.16(16.43 \sim 25.34)$ & $22.63(17.40 \sim 27.99)$ & 0.040 \\
\hline \multicolumn{4}{|l|}{ Cytokines, pg/ml } \\
\hline Interleukin 2 (IL-2) & $2.6(1.7 \sim 3.7)$ & $2.5(1.8 \sim 3.5)$ & 0.883 \\
\hline IL-4 & $2.8(2.2 \sim 3.4)$ & $2.9(2.2 \sim 3.5)$ & 0.929 \\
\hline IL-6 & $9.7(4.7 \sim 24.9)$ & $34.2(14.3 \sim 87.3)$ & 0.000 \\
\hline IL-10 & $4.2(3.1 \sim 6.1)$ & $6.7(4.7 \sim 10.0)$ & 0.000 \\
\hline Tumor necrosis factor alpha (TNF- $\alpha$ ) & $2.9(2.0 \sim 4.1)$ & $3.0(1.9 \sim 4.7)$ & 0.866 \\
\hline Interferon gamma (IFN- - ) & $7.9(4.6 \sim 12.2)$ & $16.3(8.3 \sim 49.3)$ & 0.000 \\
\hline
\end{tabular}

Data are presented as the median $\left(25^{\text {th }}-75^{\text {th }}\right.$ percentile).

doi:10.1371/journal.pone.0156465.t002

difference in the incidence of necrotizing pneumonia did not reach statistical significance $(\mathrm{P}>0.05)$.

Concerning the clinical course, we found that the median length of stay days was 9.0 (7.0 11.5) days in the RMPP group and $6.0(4.0 \sim 7.0)$ days in the GMPP group $(\mathrm{P}<0.01)$, and the median length of fever was 13.0 (11.0 16.0) days in the RMPP group versus 7.0 (3.3 10.0) days in the GMPP group $(\mathrm{P}<0.01)$ (Table 1$)$. All patients were treated with antibiotics. The median length of antibiotic therapy was 16 (14 18) days in RMPP group and 11 (8 13) days in the GMPP group, with a significant difference $(\mathrm{P}<0.01)$. Additionally, the proportion of patients required oxygentherapy and intensive care unit in the RMPP group was higher than that in the GMPP group $(\mathrm{P}<0.01)$.

Table 3. Radiological features of GMPP and RMPP patients.

\begin{tabular}{lccc}
\hline \multicolumn{1}{c}{ Radiological features } & GMPP (n= 489) & RMPP (n= 145) & P-value \\
\hline \% Patients with large lesions & $168(34.4 \%)$ & $105(72.4 \%)$ & 0.000 \\
\% Patients with pulmonary complications & & & \\
\hline Pleural effusion & $54(11.0 \%)$ & $70(48.3 \%)$ & 0.000 \\
Lobar atelectasis & $54(11.0 \%)$ & $38(26.2 \%)$ & 0.000 \\
Pulmonary consolidation & $19(3.9 \%)$ & $32(22.1 \%)$ & 0.000 \\
Pleural thickening & $17(3.5 \%)$ & $17(11.7 \%)$ & 0.000 \\
Necrotizing pneumonia & $2(0.4 \%)$ & $2(1.4 \%)$ & 0.226 \\
\hline
\end{tabular}

Data are presented as number (percentage).

doi:10.1371/journal.pone.0156465.t003 
Table 4. Predictive values of the independent correlation factors in patients with RMPP.

\begin{tabular}{|c|c|c|c|c|c|}
\hline Independent factors & Cutoff value & Sensitivity & Specificity & AUC & P-value \\
\hline Age, years & 3.875 & 0.745 & 0.564 & 0.674 & 0.000 \\
\hline Neutrophil, \% & 68.6 & 0.684 & 0.811 & 0.803 & 0.000 \\
\hline C-reactive protein (CRP), mg/L & 16.5 & 0.747 & 0.772 & 0.817 & 0.000 \\
\hline Lactatedehydrogenase (LDH), IU/L & 417 & 0.797 & 0.650 & 0.772 & 0.000 \\
\hline Prealbumin (PAB), g/L & 0.095 & 0.757 & 0.637 & 0.747 & 0.000 \\
\hline Immunoglobulin A (IgA), g/L & 0.66 & 0.861 & 0.402 & 0.636 & 0.000 \\
\hline $\mathrm{CD}_{8}^{+}, \%$ & 25.05 & 0.430 & 0.768 & 0.603 & 0.006 \\
\hline Interleukin 6 (IL-6), pg/ml & 14.75 & 0.835 & 0.634 & 0.758 & 0.000 \\
\hline IL-10, pg/ml & 4.65 & 0.759 & 0.610 & 0.722 & 0.000 \\
\hline Interferon gamma (IFN- - ), pg/ml & 15.50 & 0.557 & 0.846 & 0.724 & 0.000 \\
\hline
\end{tabular}

AUC: area under the ROC curve; Cut-off value: the value on the ROC curve is closest to the upper right to take maximum sensitivity and specificity; Pvalue: the AUC value of the independent factors compared to ROC curve reference value 0.5 .

doi:10.1371/journal.pone.0156465.t004

Table 5. Stepwise logistic regression analysis for the related factors predicting the RMPP.

\begin{tabular}{|c|c|c|c|c|c|c|c|}
\hline \multirow[t]{2}{*}{ Variable } & \multirow[t]{2}{*}{ B } & \multirow[t]{2}{*}{ S.E. } & \multirow[t]{2}{*}{ Wald } & \multirow[t]{2}{*}{ P-value } & \multirow[t]{2}{*}{ OR } & \multicolumn{2}{|c|}{$95 \% \mathrm{Cl}$} \\
\hline & & & & & & Lower & Upper \\
\hline C-reactive protein (CRP) $\geq 16.5 \mathrm{mg} / \mathrm{L}$ & 0.705 & 0.330 & 4.570 & 0.033 & 2.023 & 1.060 & 3.861 \\
\hline Lactatedehydrogenase (LDH) $\geq 417 \mathrm{IU} / \mathrm{L}$ & 0.782 & 0.319 & 5.998 & 0.014 & 2.185 & 1.169 & 4.084 \\
\hline Interleukin 6 (IL-6) $\geq 14.75 \mathrm{pg} / \mathrm{ml}$ & 0.839 & 0.333 & 6.341 & 0.012 & 2.314 & 1.204 & 4.446 \\
\hline
\end{tabular}

doi:10.1371/journal.pone.0156465.t005

\section{Predictive values of the independent correlation factors in patients with RMPP}

To explore the predictive values of laboratory date for RMPP, receiver operator characteristic (ROC) curves were made and the cut-off values with maximum sensitivities and specificities were determined. Analysis of these ROC curves showed that the percentage of neutrophil, CRP, LDH, PAB, IL-6, IL-10 and IFN- $\gamma$ were useful for differentiating patients with RMPP from those with GMPP (Table 4). When the cut-off values for the percentage of neutrophil, CRP, LDH, PAB, IL-6, IL-10 and IFN- $\gamma$ were set at $68.6 \%, 16.5 \mathrm{mg} / \mathrm{L}, 417 \mathrm{IU} / \mathrm{L}, 0.095 \mathrm{~g} / \mathrm{L}$, $14.75 \mathrm{pg} / \mathrm{ml}, 4.65 \mathrm{pg} / \mathrm{ml}$ and $15.50 \mathrm{pg} / \mathrm{ml}$, respectively, the sensitivity and specificity in differentiating RMPP from GMPP were $68.4 \%$ and $81.1 \%, 74.7 \%$ and $77.2 \%, 79.7 \%$ and $65.0 \%, 75.7 \%$ and $63.7 \%, 83.5 \%$ and $63.4 \%, 75.9 \%$ and $61.0 \%, 55.7 \%$ and $84.6 \%$, respectively.

\section{Multiple logistic regression analysis for the related factors predicting the RMPP}

Multiple logistic regression analysis of 634 cases was performed to assess predictors which allowed the differential diagnosis of RMPP and GMPP. The CRP $\geq 16.5 \mathrm{mg} / \mathrm{L}, \mathrm{LDH} \geq 417 \mathrm{IU} / \mathrm{L}$ and IL- $6 \geq 14.75 \mathrm{pg} / \mathrm{ml}$ were significantly predictive regarding the differentiation between the two groups, with the odd ratio (OR) values of $2.023,2.185$, and 2.314 , respectively in Table 5 .

\section{Discussion}

$\mathrm{MP}$ is one of the major pathogens causing CAP in children, especially in Asian. Although MP infection was traditionally thought to be a self-limited process, more and more severe cases 
even fatal cases of MP infections were reported in recent years [4-11]. Cases of RMPP, which display clinical and radiological progression after macrolide therapy for 7 days or longer, are reported increasingly $[13,19,20]$. So it is essential for pediatricians to recognize RMPP early, treat it promptly and prevent the progress of the disease. To our knowledge, there is still a paucity of data about clinical characteristics and risk factor of RMPP in a large number of cases until now.

In the present, retrospective, observational study, 634 patients with MPP were enrolled, and the different clinical characteristics between the RMPP patients and GMPP patients were compared. According to the diagnostic criteria of RMPP [12,13], 145 cases were diagnosed as RMPP, while 489 were GMPP. Firstly, we found that the median age of the RMPP patients was much older than that of the GMPP patients (5.9 (3.8 8.0) versus 3.4 (1.9 6.3), $\mathrm{P}<0.01$ ), which was in accordance with the previous reports $[20,21]$. The immune system in older children was relatively more mature than that in younger children, and inappropriate immune response to MP was easy to produce excessive inflammatory reaction, which might contribute to the development of RMPP.

Secondly, more severe signs and symptoms and higher incidence of extra-pulmonary complications were found in the RMPP group than those in the GMPP group $(\mathrm{P}<0.05)$. We also found that patients with RMPP showed longer median length of stay days, longer median length of fever days and longer median length of antibiotic therapy days than those of patients with GMPP $(\mathrm{P}<0.01)$. Moreover, the proportion of patients required oxygen therapy and intensive care unit in the RMPP group was higher than that in the GMPP group $(\mathrm{P}<0.01)$. These results implied that RMPP was refractory to treatment and could result in prolonged clinical course.

Additionally, our study showed that $72.4 \%$ of the patients in the RMPP group had large lesions of radiological manifestation versus $34.4 \%$ in the GMPP group $(\mathrm{P}<0.01)$. And higher incidence of pleural effusion, lobar atelectasis, consolidation and pleural thickening were found in the RMPP group than in the GMPP group $(\mathrm{P}<0.01)$. The diversification of imaging findings might due to direct microbe effect and host strong immune response and these significant radiographical evidences of lung damage were in compliance with the complicated course.

Although the underlying mechanisms are still uncertain, the macrolide-resistant MP infection and excessive immunological inflammation are most commonly proposed [22-26]. Cao et al [27] reported that the resistance rate of MP in adult patients with respiratory tract infection was reached to $69 \%$ in 2010. But in our previous study we found higher prevalence (87.7\%) of macrolide-resistance in MPP patients, and there were no significant difference of resistance rate of MP between the GMPP group and the RMPP group [17]. So we think that cell-mediated immunological response plays an important role in the progression of MPP. Tanaka et al. demonstrated that the levels of serum interleukin-18, which promotes Th1 cytokine responses, in patients with severe MP were higher than those in mild cases [21]. Here, we compared some other laboratory markers between the RMPP group and GMPP group. Several serum characteristics including markedly increased CRP levels, LDH levels and percentage of neutrophils were observed to be associated with RMPP, which was well consistent with other reports [20, 28-30]. Additionally, our study firstly demonstrated a significant lower level of PAB in the RMPP patients, which perhaps also indicated severe systemic inflammatory response to MP infection.

Inflammatory cytokines were also involved in the immunopathogenesis of MP infection [31-33]. In our study, we found that higher levels of IL-6, IL-10, IFN- $\gamma$ in the RMPP group than those in the GMPP group $(\mathrm{P}<0.01)$. Meanwhile, higher levels of IgA and higher percentage of $\mathrm{CD}^{+}$in the RMPP patients were detected when compared with the GMPP group $(\mathrm{P}<0.01, \mathrm{P}<0.05$, respectively). The excessive inflammation reaction may lead to release of cytokines and immune disorder, which is might related to the severity of RMPP in children. 
To investigate potential factors that may allow differentiation between RMPP and GMPP, we analyzed age and some laboratory markers, which were significantly different between the two groups using ROC curve. In our study, we found that the area under the curve for seven independent factors, including the percentage of neutrophil, CRP, LDH, PAB, IL-6, IL-10 and IFN- $\gamma$ were above 0.7 in ROC curve analysis, indicating fair discriminative power for predicting RMPP. The optimal cutoff value for these seven factors was $68.6 \%, 16.5 \mathrm{mg} / \mathrm{L}, 417 \mathrm{IU} / \mathrm{L}, 0.095 \mathrm{~g} /$ $\mathrm{L}, 14.75 \mathrm{pg} / \mathrm{ml}, 4.65 \mathrm{pg} / \mathrm{ml}$ and $15.50 \mathrm{pg} / \mathrm{ml}$, respectively. Furthermore, basing on the cutoff values of these seven factors, multiple logistic regression analysis were made to improve the predicted accuracy. We found that the LDH $\geq 417 \mathrm{IU} / \mathrm{L}, \mathrm{CRP} \geq 16.5 \mathrm{mg} / \mathrm{L}$ and IL- $6 \geq 14.75 \mathrm{pg} / \mathrm{ml}$ were significant predictors of RMPP. LDH was associated with many pulmonary diseases, such as obstructive diseases, microbial pulmonary diseases, and interstitial lung diseases [34, 35]. Several studies $[21,29,30]$ also found that serum LDH was elevated in RMPP. In our study, we found that the area under the curve for LDH was 0.772 in ROC curve analysis, indicating fair discriminative power for predicting RMPP. The optimal cutoff for LDH was 417 IU/L, with a sensitivity of $79.7 \%$ and specificity of $65.0 \%$, which was similar with previous repot [29]. And stepwise logistic regression analyzed that the serum LDH (odds ratio of $2.185,95 \% \mathrm{CI}$ 1.169 4.084, $\mathrm{P}=0.014$ ) was significant meaningful. CRP is a gross biochemical index of inflammation and is used commonly in the clinical setting. CRP reflected the acute severe systemic inflammatory reactions to MP infection, and was suggestive of a well-developed immune system. Liu et al showed that the cutoff value of CRP for RMPP was $40 \mathrm{mg} / \mathrm{L}$ [30]. The cutoffs were greater than in our study. The main reason may be that their results were obtained from a small case series, and they had more serious illnesses. In this study, the optimal cutoff point for $\mathrm{CRP}$ was $16.5 \mathrm{mg} / \mathrm{L}$, with a sensitivity of $74.7 \%$ and specificity of $77.2 \%$, and the odds ratio of logistic regression analysis was 2.023 . These indicate theirs clinical utility in identifying patients at high risk for RMPP. More interestingly, this is the first report demonstrated that IL-6 $\geq 14.75 \mathrm{pg} / \mathrm{ml}$ might help us to differentiate RMPP from GMPP. However, the measurement of IL-6 is relatively costly and has a relatively high inter-laboratory variation in accuracy of the measure. So IL-6 is not a parameter commonly measured in the clinical setting but limited to research purposes.

This study has several limitations. Firstly, it was a retrospective study, and therefore there may have been some selection bias. Secondly, there may be some cases in which the patients had a combined MP and other pathogens infection which cannot be detected, which might result in RMPP. Thirdly, the distribution of patients between the two groups is not matching, which might affect the statistic results. Fourthly, in spite of the potential usefulness of using a biochemical parameter for categorizing RMPP from MPP patients, the sensitivity and the specificity of the variables identified are relatively modest. Therefore a large number of patients with RMPP are needed to be enrolled and a further prospective study is needed to be carried out.

In conclusion, older children are more prone to developing RMPP, leading to more severe presentations, higher incidence of extra-pulmonary complications and more serious radiological findings. Clinician might use the LDH, CRP and/or IL- 6 cut off (CRP $\geq 16.5 \mathrm{mg} / \mathrm{L}, \mathrm{LDH}$ $\geq 417 \mathrm{IU} / \mathrm{L}$ and IL- $6 \geq 14.75 \mathrm{pg} / \mathrm{ml}$ ) for identifying children at higher risk of RMPP in comparison to all the other clinical, radiological and biochemical information.

\section{Supporting Information}

S1 File. STROBE Statement-Checklist of items that should be included in reports of casecontrol studies.

(DOC) 


\section{Acknowledgments}

We are grateful to colleagues in Department of Pulmonology for taking care of patients.

\section{Author Contributions}

Conceived and designed the experiments: YYZ ZMC. Performed the experiments: YYZ YLZ. Analyzed the data: YYZ SXL. Contributed reagents/materials/analysis tools: DHY XLW. Wrote the paper: YYZ. Critical revision of the manuscript for important intellectual content: ZMC.

\section{References}

1. Michelow IC, Olsen K, Lozano J, Rollins NK, Duffy LB, Ziegler T, et al. Epidemiology and clinical characteristics of community-acquired pneumonia in hospitalized children. Pediatrics 2004; 113(4):701707. PMID: 15060215

2. Defilippi A, Silvestri M, Tacchella A, Giacchino R, Melioli G, Di Marco E, et al. Epidemiology and clinical features of Mycoplasma pneumoniae infection in children. Resp Med 2008; 102(12):1762-1768.

3. Waites K. New concepts of Mycoplasma pneumoniae infections in children. Pediatr Pulmonol 2003; 36 (4):267-78. PMID: 12950038

4. Kim CK, Kim SW, Kim JS, Koh YY, Cohen AH, Deterding RR, et al. Bronchiolitis obliterans in the 1990s in Korea and the United States. Chest 2001; 120(4):1101-6. PMID: 11591545

5. Wang RS, Wang SY, Hsieh KS, Chiou YH, Huang IF, Cheng MF, et al. Necrotizing pneumonitis caused by Mycoplasma pneumoniae in pediatric patients: report of five cases and review of literature. Pediatr Infect Dis J 2004; 23(6):564-7. PMID: 15194841

6. Socan M, Ravnik I, Bencina D, Dovc P, Zakotnik B, Jazbec J. Neurological symptoms in patients whose cerebrospinal fluid is culture- and/or polymerase chain reaction-positive for Mycoplasma pneumoniae. Clin Infect Dis 2001; 32(2):E31-5. PMID: 11170938

7. Azumagawa K, Kambara $\mathrm{Y}$, Murata $\mathrm{T}$, Tamai $\mathrm{H}$. Four cases of arthritis associated with Mycoplasma pneumoniae infection. Pediatr Int 2008; 50(4):511-3. doi: 10.1111/j.1442-200X.2008.02622.x PMID: 19143974

8. Hwkins S, Rausch CM, McCanta AC. Constrictive pericarditis secondary to infection with Mycoplasma pneumoniae. Curr Opin Pediatr 2011; 23(1):126-9. PMID: 21107263

9. Khan FY, Ayassin M. Mycoplasma pneumoniae associated with severe autoimmune hemolytic anemia: case report and literature review. Braz J Infect Dis 2009; 13(1):77-9. PMID: 19578637

10. Radisic M, Torn A, Gutierrez P, Defranchi HA, Pardo P. Severe acute lung injury caused by Mycoplasma pneumoniae: potential role for steroid pulses in treatment. Clin Infect Dis 2000; 31(6):1507-11. PMID: 11096025

11. Takiguchi $Y$, Shikama N, Aotsuka N, Koseki H, Terano T, Hirai A. Fulminant Mycoplasma pneumoniae pneumonia. Intern Med 2001; 40(4):345-8. PMID: 11334397

12. The Subspecialty Group of Respiratory Disease, The Society of Pediatrics, Chinese Medical Association, The Editorial Board, Chinese Journal of Pediatrics. Guidelines for management of community acquired pneumonia in children (the revised edition of 2013) (I). Chin J Pediatr 2013; 51(10):745-749.

13. Tamura A, Matsubara K, Tanaka T, Nigami H, Yura K, Fukaya T. Methylprednisolone pulse therapy for refractory Mycoplasma pneumoniae pneumonia in children. J Infect 2008; 57(3):223-228. doi: 10. 1016/j.jinf.2008.06.012 PMID: 18656264

14. Nelson S, Belknap SM, Carlson RW, Dale D, Deboisblanc B, Farkas S, et al. A randomized controlled trial of filgrastim as an adjunct to antibiotics for treatment of hospitalized patients with communityacquired pneumonia, CAP Study Group. J Infect Dis 1998; 178(4):1075-80. PMID: 9806037

15. Suzuko U, Keisuke S, Hiroyuki E, Kazunobu O, Kenji O, Tomomichi K, et al. Japanese Guidelines for the Management of Respiratory Infectious Diseases in Children 2007 with focus on pneumonia. Pediatrics International 2011; 53(2): 264-276. PMID: 21648118

16. Suzuki S, Yamazaki T, Narita M, Okazaki N, Suzuki I, Andoh T, et al. Clinical evaluation of macrolideresistant Mycoplasma pneumoniae. Antimicrob Agents Chemother 2006; 50(2):709-712. PMID: 16436730

17. Zhou Y, Zhang Y, Sheng Y, Zhang L, Shen Z, Chen Z. More complications occurr in macrolide-resistant than in macrolide-sensitive Mycoplasma pneumoniae pneumonia. Antimicrob Agents Chemother 2014; 58(2):1034-8. doi: 10.1128/AAC.01806-13 PMID: 24277047 
18. Tan TQ, Mason EO Jr, Wald ER, Barson WJ, Schutze GE, Bradley JS, et al. Clinical characteristics of children with complicated pneumonia caused by Streptococcus pneumonia. Pediatrics 2002; 110 (1Pt1):1-6.

19. Luo Z, Luo J, Liu E, Xu X, Liu Y, Zeng F, et al. Effects of prednisolone on refractory Mycoplasma pneumoniae pneumonia in children. Pediatr Pulmonol 2014; 49(4):377-80. doi: 10.1002/ppul.22752 PMID: 23401275

20. Wang M, Wang Y, Yan Y, Zhu C, Huang L, Shao X, et al. Clinical and laboratory profiles of refractory Mycoplasma pneumoniae pneumonia in children. Int $\mathrm{J}$ Infect Dis 2014; 29:18-23. doi: 10.1016/.ijid. 2014.07.020 PMID: 25449230

21. Lee KY, Lee HS, Hong JH, Lee MH, Lee JS, Burgner D, et al. Role of prednisolone treatment in severe Mycoplasma pneumoniae pneumonia in children. Pediatr Pulmonol 2006; 41(3):263-8. PMID: 16437541

22. Tanaka H, Narita M, Teramoto S, Saikai T, Oashi K, Igarashi T, et al. Role of interleukin-18 and T-helper type 1 cytokines in the development of Mycoplasma pneumoniae pneumonia in adults. Chest 2002; 121(5):1493-7. PMID: 12006434

23. Sekine $\mathrm{H}$, Taguchi $\mathrm{H}$, Watanabe $\mathrm{H}$, Kawai $\mathrm{S}$, Fujioka $\mathrm{Y}$, Goto $\mathrm{H}$, et al. Immunological analysis and pathological examination of gnotobiotic mice monoassociated with Mycoplasma pneumoniae. J Med Microbiol 2009; 58(Pt 6):697-705. doi: 10.1099/jmm.0.007872-0 PMID: 19429744

24. Waites KB, Balish MF, Atkinson TP. New insights into the pathogenesis and detection of Mycoplasma pneumoniae infections. Future Microbiol 2008; 3(6):635-48. doi: 10.2217/17460913.3.6.635 PMID: 19072181

25. Fernald GW. Immunologic mechanisms suggested in the association of $M$. pneumoniae infection and extrapulmonary disease: a review. Yale J Biol Med 1983; 56(5-6):475-479. PMID: 6433568

26. Waites KB, Talkington DF. Mycoplasma pneumoniae and its role as a human pathogen. Clin Microbiol Rev 2004; 17(4):697-728. PMID: 15489344

27. Cao B, Zhao CJ, Yin YD, Zhao F, Song SF, Bai L, et al. High prevalence of macrolide resistance in Mycoplasma pneumoniae isolates from adult and adolescent patients with respiratory tract infection in China. Clin Infect Dis 2010; 51(2):189-194. doi: 10.1086/653535 PMID: 20540621

28. Oishi T, Narita M, Matsui K, Shirai T, Matsuo M, Negishi J, et al. Clinical implications of interleukin-18 levels in pediatric patients with Mycoplasma pneumoniae pneumonia. J Infect Chemother 2011; 17 (6):803-6. doi: 10.1007/s10156-011-0265-7 PMID: 21681500

29. Inamura N, Miyashita N, Hasegawa S, Kato A, Fukuda Y, Saitoh A, et al. Management of refractory Mycoplasma pneumoniae pneumonia: utility of measuring serum lactate dehydrogenase level. J Infect Chemother 2014; 20(4):270-3. doi: 10.1016/j.jiac.2014.01.001 PMID: 24486173

30. Liu JR, Peng Y, Yang HM, Li HM, Zhao SY, Jiang ZF. Clinical characteristics and predictive factors of refractory Mycoplasma pneumoniae pneumonia. Zhonghua Er Ke Za Zhi. 2012; 50(12):915-8. PMID: 23324149

31. Narita M, Tanaka H. Cytokines involved in the severe manifestations of pulmonary diseases caused by Mycoplasma pneumonia. Pediatr Pulmonol 2007; 42(4):397. PMID: 17330264

32. Hardy RD, Jafri HS, Olsen K, Wordemann M, Hatfield J, Rogers BB, et al. Elevated cytokine and chemokine levels and prolonged pulmonary airflow resistance in a murine Mycoplasma pneumoniae pneumonia model: a microbiologic, histologic, immunologic, and respiratory plethysmographic profile. Infect Immun 2001; 69(6):3869-76. PMID: 11349053

33. Yang J, Hooper WC, Phillips DJ, Talkington DF. Regulation of proinflammatory cytokines in human lung epithelial cells infected with Mycoplasma pneumoniae. Infect Immun 2002; 70(7):3649-55. PMID: 12065506

34. Drent M, Cobben NA, Henderson RF, Wouters EF, van Dieijen- Visser M. Usefulness of lactate dehydrogenase and its isoenzymes as indicators of lung damage or inflammation. Eur Respir J 1996; 9 (8):1736-1742. PMID: 8866602

35. Nakajima M, Kawahara Y, Yoshida K, Miyashita N, Niki Y, Matsushima T. Serum IL-6 as a possible marker for amiodarone induced pulmonary toxicity. Intern Med 2000; 39(12):1097-1100. PMID: 11197799 